Review

\title{
The Role of Colonic Bacteria in the Metabolism of the Natural Isoflavone Daidzin to Equol
}

\section{Fatemeh Rafii}

Division of Microbiology, National Center for Toxicological Research, FDA, Jefferson, AR 72079, USA; E-Mail: Fatemeh.Rafii@fda.hhs.gov; Tel.: +1-870-543-7342; Fax: +1-870-543-7307

Academic Editor: Wendy Roslyn Russell

Received: 7 November 2014 / Accepted: 7 January 2015 / Published: 14 January 2015

\begin{abstract}
Isoflavones are found in leguminous plants, especially soybeans. They have a structural similarity to natural estrogens, which enables them to bind to estrogen receptors and elicit biological activities similar to natural estrogens. They have been suggested to be beneficial for the prevention and therapy of hormone-dependent diseases. After soy products are consumed, the bacteria of the intestinal microflora metabolize isoflavones to metabolites with altered absorption, bioavailability, and estrogenic characteristics. Variations in the effect of soy products have been correlated with the isoflavone metabolites found in plasma and urine samples of the individuals consuming soy products. The beneficial effects of the soy isoflavone daidzin, the glycoside of daidzein, have been reported in individuals producing equol, a reduction product of daidzein produced by specific colonic bacteria in individuals called equol producers. These individuals comprise $30 \%$ and $60 \%$ of populations consuming Western and soy-rich Asian diets, respectively. Since the higher percentage of equol producers in populations consuming soy-rich diets is correlated with a lower incidence of hormone-dependent diseases, considerable efforts have been made to detect the specific colonic bacteria involved in the metabolism of daidzein to the more estrogenic compound, equol, which should facilitate the investigation of the metabolic activities related to this compound.
\end{abstract}

Keywords: metabolites; metabolism; intestinal bacteria; phytoestrogen; isoflavone; daidzin; daidzein; equol 


\section{Introduction}

The community of microorganisms residing in the human gastrointestinal tract forms a complex ecosystem populated with over $10^{11}-10^{12}$ bacterial cells per gram of contents of the large intestine [1-3]. The colon harbors more than 400 bacterial species, the majority of which are anaerobes; they are present both in the colonic contents and attached to the mucosa [4]. The diverse community of microorganisms in the colon has coevolved with the human host, with which it has a mutually beneficial relationship $[1,3,5]$. The colonic environment provides nutrients, $\mathrm{pH}$, temperature, and redox potential favorable for the biological and biochemical reactions of the microbial community [4]. The microbial community acts as a barrier against pathogenic bacteria that might colonize the colon, and it metabolizes nutrients, drugs, and xenobiotics. Microbial metabolism by colonic bacteria is reported to contribute to the metabolic, nutritional, physiological, and immunological processes of the host; the extent of this contribution to human health is unknown [2].

The collective genome of the human intestinal microbiota is comprised of 3.3 million genes, approximately 150 times more genes than are in the human genome [2]. The metabolome, or the metabolic signature of an individual, is dependent not only upon the host but also upon the interaction of the host with the microflora $[2,3,5,6]$. Some of the metabolites found in a host are exclusively the result of the activities of bacteria in the colonic microbial community. This includes the digestion of fibers not metabolized by host enzymes into short-chain fatty acids, and the metabolism of isoflavones, which is performed by a number of bacteria [2,3,5]. The types of colonic bacteria metabolizing isoflavones in each individual affect the outcome of the consumption of isoflavones [2,3]. Inter-subject differences in response to isoflavones may be due to specific differences in gut microflora species [7-16]. In this mini-review, the role of colonic bacteria in the metabolism of the isoflavone daidzin will be discussed.

\section{Isoflavone Phytoestrogens}

Isoflavone phytoestrogens are bioactive plant constituents that are found, mainly in conjugated forms, in the products of more than 40 plant species, including soybeans, other legumes, whole grains, berries, and nuts [12,17-21]. Two of the widely studied isoflavones from soybeans are daidzin and genistin, the glycoside forms of daidzein and genistein, respectively. They are found in different concentrations depending on the growth conditions [21]. Among other naturally occurring isoflavonoids from legumes are biochanin A, formononetin, and glycitein, the methoxylated forms of genistein, daidzein, and 6,7,4'-trihydroxyisoflavone, respectively [22].

Although isoflavones are nonsteroidal, they have similarities in chemical structure to mammalian estrogens, show estrogenic activities in biological assays, and induce estrogen-like effects in mammalian systems [23-27]. In legume plants, daidzin and genistin act as signal molecules for symbiotic nitrogen-fixing bacteria [28]. In mammalian systems, they have numerous biological activities and may also be metabolized, which alters their effects $[29,30]$.

\section{Role of Dietary Isoflavones}

The soy-rich diets of Asian populations contain high levels of isoflavones, which are believed to contribute to the lower incidence of hormone-dependent diseases, cardiovascular diseases, breast, prostate 
and colon cancer, and menopausal symptoms, including osteoporosis [12,29,31-36]. The rate of breast cancer in Asian women is one third to one half of that in Caucasians [29] and the frequency of menopausal hot flashes in Asian women is less than that of Western women [11,32-34,37]. Adoption of Western diets by immigrant Asians has put them at higher risk of breast and prostate cancer, suggesting that dietary and environmental factors are more important than racial characteristics [38]. The beneficial effects of dietary isoflavones in soy are not always clear from in vivo studies [29], but health benefits have been reported in more than half of the clinical studies [16,39].

Variations observed in the effects of phytoestrogens depend on various factors, including differences in the population of bacteria in the intestinal tract capable of metabolizing isoflavones. Observation and interventional studies have shown the ability of the intestinal microfloras of some individuals to convert isoflavones to metabolites that may result in a reduced risk of hormone-dependent diseases [12,39-47].

\section{Role of Intestinal Microflora in the Bioavailabilities of Isoflavones}

\subsection{Intestinal Bacteria}

Bacteria in both the small and the large intestine are involved in the metabolism of natural isoflavones [18,22,48-50]. The microbial hydrolytic enzymes are involved in the deconjugation of natural isoflavones (primary metabolism) and release of more bioavailable products. The bacteria converting primary metabolites to more or less estrogenic secondary metabolites are mainly found in the colon $[18,22,31,36,48-50]$.

\subsection{Hydrolyis of Isoflavones}

The intestinal microflora has a major role in the metabolism, bioavailability, biological activities, and metabolomic profiles of dietary isoflavones. Soybeans contain the natural isoflavone glycosides, daidzin and genistin, which because of their large hydrophilic structures are not easily absorbed across the enterocytes or intestinal absorptive cells [18,22,31,36,48-52]. However, intestinal bacteria produce hydrolytic enzymes capable of hydrolyzing the natural isoflavones and releasing the unconjugated isoflavones, daidzein and genistein, which are more estrogenic and readily absorbed [31,49,51-55]. In addition to the glucosidase produced from the intestinal brush border [56], $\beta$-glucosidases of bacteria in the small intestine have a high affinity for daidzin and genistin, hydrolyzing them to their aglycones. Deglycosylation of these compounds is an important step in the absorption, metabolism, excretion, and biological activities of isoflavones [54,56].

After daidzein and genistein enter the circulation via absorption through the intestine, they may be transported to the liver, where they are conjugated by the liver enzymes to form sulfated, glycosylated and glucuronidated isoflavones, which are more water-soluble [57]. Gu et al. [57] showed that isoflavones in human plasma are predominantly glucuronides (75\%), sulfates (24\%), and aglycones (1\%).

\subsection{Metabolism of Isoflavones by Colonic Bacteria}

Metabolism of isoflavones by the colonic microflora alters their biological activities [31,54,58-62]. The liver conjugates of isoflavones may be excreted in the bile and pass into the intestinal lumen $[56,63]$. These isoflavones, along with ingested and deconjugated isoflavones in the small intestine, enter the 
colon, where they are subjected to further hydrolysis and microbial transformation by the colonic microflora [56,63].

Enzymes from the human colonic microflora deconjugate conjugated isoflavones, which are then reabsorbed through the enterohepatic circulation, altering their pharmacokinetic distribution, or metabolize them further to compounds that are either more or less estrogenic than the parent compounds (Figure 1) [29,64]. Thus, the host colonic bacteria are important in the bioavailabilities and biological activities of isoflavones and influence the metabolomic profile of the host after consumption of isoflavones $[64,65]$. Some of the intestinal bacterial strains that are involved in the metabolism of the isoflavone daidzin (Figure 1) have been identified and their roles in the production of primary and secondary metabolites are described in the following sections.

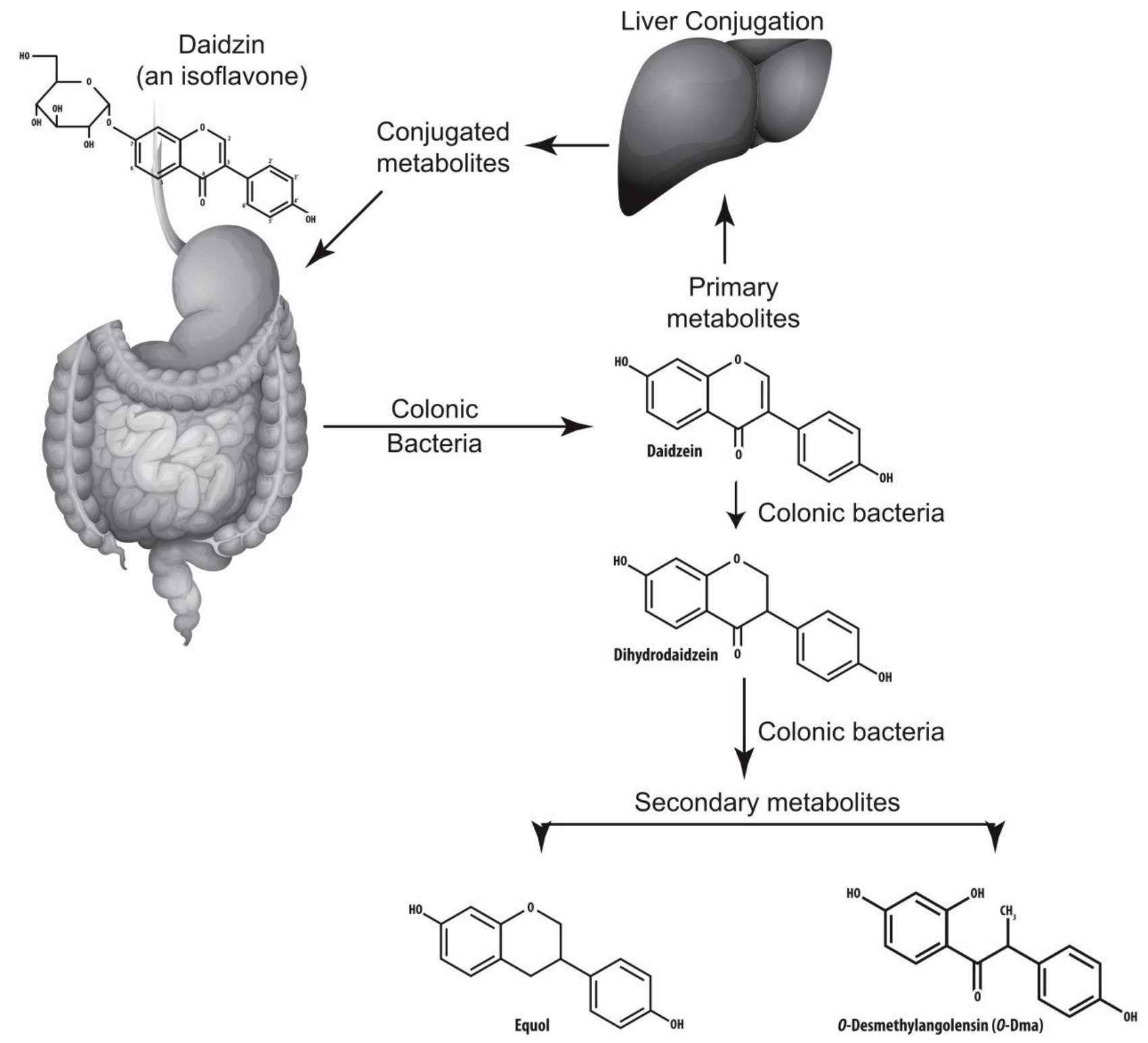

Figure 1. Illustration of the role of colonic bacteria in the production of primary and secondary metabolites from the isoflavone daidzin and in the metabolism and circulation of daidzein liver conjugates. Whereas the intestinal microflora produces $(S)$-equol from daidzein, the $O$-desmethylangolensin (DMA) produced as the result of intestinal bacterial activities is $(R)$ - $O$-DMA. 
One of the widely studied metabolites produced by bacteria residing in the gut of subjects consuming soy-containing isoflavones is equol, which until recently was thought to be produced only by anaerobic colonic bacteria [31,54]. Only $30 \%$ of the western population carries colonic bacteria that convert daidzein to equol. This population, which is designated as equol producers $[7,13,15,64-66]$, may benefit from consumption of soy products more than non-producers, which may convert daidzein to a less estrogenic metabolite, $O$-desmethylangolensin $(O$-DMA) [29]. The serum equol concentration in nonproducers is $\leq 40 \mathrm{nmol} / \mathrm{L}$, which is much lower than the $\geq 83 \mathrm{nmol} / \mathrm{L}$ found in the serum of equol producers [36]. The presence of $60 \%$ equol producers in Asian populations, versus the $30 \%$ in Western populations, is associated with a lower incidence of hormone-dependent diseases among this population that also consumes more dietary isoflavones through a soy-based diet $[29,54,55]$. In the following sections, equol, the biologically important metabolite of daidzein, and the intestinal bacteria involved in the metabolism of natural isoflavones and biosynthesis of equol are discussed.

\section{Equol}

One of the most widely studied metabolites of the isoflavone daidzein is equol, (3S)-3-(4-hydroxyphenyl)7-chromanol, which is produced by bacteria that have specialized enzymes [7,14,16,29,54,55]. The structure has two phenolic rings, with reactive hydroxyl groups, and a central furan ring with an inert oxygen (Figure 1). It is insoluble in water and acid-labile. Synthetic equol exists as two enantiomers, $(S)-(-)$-equol and $(R)-(+)$-equol, both of which are biologically active and modulate androgen action, but only $S$-equol occurs naturally [54].

$S$-Equol is produced by the action of intestinal bacteria on daidzein in $20 \%-30 \%$ of the population in Western countries and 50\%-60\% of Asian populations consuming soy-containing diets [54,55]. Because of its enhanced ability to bind to estrogen receptors, equol is one of the most bioactive isoflavones [9,23,24,45,47,54,55,67-72]. After consumption of a diet containing soy, equol is found as a metabolic by-product of the activity of colonic intestinal bacteria in human equol producers $[7,36,54,55,66]$.

Equol has an affinity for the human estrogen receptors $\operatorname{ER} \alpha$ and $\operatorname{ER} \beta$, strong antioxidant activities, and other properties [9,36,69,70,73-77]. Equol is readily absorbed through the colonic wall, but the rate of its clearance from plasma is slower than that of daidzein [15]. The production of equol in humans is solely the result of the intestinal bacterial metabolism of daidzein [36,54,55]. Daidzein, $O$-DMA, and equol bind to and activate the estrogen receptors ER $\alpha$ and ER $\beta$ so they will translocate to the nucleus and bind to the estrogen response element, a specific DNA sequence with high affinity for estrogen receptors located in or near the promoter region of target genes $[42,67]$. The binding of activated ER $\alpha$ and ER $\beta$ to the estrogen response element is the first step in the induction of gene activation and transcription [42]. The affinity of $O$-DMA and equol for ER $\alpha$ and ER $\beta$ is higher than that of daidzein [78-80]. Equol is able to activate the binding of both $\operatorname{ER} \alpha$ and $\operatorname{ER} \beta$ to the estrogen response element better than daidzein, which preferentially activates binding of ER $\beta$ to the estrogen response element [81].

Markiewicz et al. [78] showed that equol was more estrogenic for human adenocarcinoma cells than daidzein. Both $O$-DMA and equol stimulate the growth of estrogen-responsive MCF-7 breast cancer cells more than daidzein [31]. It has been shown that equol has higher antioxidant activity than daidzein $[9,36,69,73,75-77,82-85]$. Studies have indicated that equol is better than daidzein for inhibiting 
the proliferation of benign and malignant prostatic cell lines [86]. Equol is 100 times stronger than daidzein in stimulating the mRNA expression of an estrogen-responsive protein (pS2) in MCF-7 cells [80]. Exposure of MCF-7 cells to both equol and 17 $\beta$-estradiol reduces the expression of pS2 mRNA below the level of expression of this gene with estradiol alone, indicating that equol has antiestrogenic activity [80]. Equol, whether produced by the microbiota or directly administered to female mice, is also anti-estrogenic in the reproductive tissue [87]. Administration of equol and endogenously produced equol was shown to reduce the expression of the progesterone receptor in the mice vaginal epithelium, indicating that equol reduces the tissue estrogen-dependent response [87].

\section{Colonic Bacteria Associated with the Metabolism of Isoflavones}

Metabolites of phytoestrogens have been detected in the urine of humans and experimental animals [15]. They are also found as the products of colonic microflora in human flora-associated-germ-free rats $[17,88]$. The levels of metabolites of isoflavones (equol and $O$-DMA ) that are found in germ-free rats after association with the colonic microflora of humans are dependent on the amounts produced by these bacteria, whether from equol producers or nonproducers $[17,88]$. Intersubject differences in responses to isoflavones may reflect subject-specific differences in gut microfloral species [89]. Chang and Nair [58,59] showed that incubation of daidzein and genistein with fecal bacteria under anaerobic conditions resulted in the conversion of genistein to dihydrogenistein and daidzein to dihydrodaidzein and equol, with equol being the major metabolite detected in the cultures.

Hur and Rafii [22] found that Eubacterium limosum, an anaerobic intestinal bacterium capable of producing O-demethylation of several methylated compounds, metabolizes three naturally occurring isoflavones, biochanin A, formononetin and glycitein. The demethylated products, genistein, daidzein and 6,7,4'-trihydroxyisoflavone, respectively, were detected in cultures after incubation with the bacterial cells but not with spent culture medium, indicating that the demethylase activities are cell-associated.

Hur et al. [48], screening the fecal bacteria from a healthy individual, found an E. coli strain, HGH21, and a Gram-positive bacterial strain, HGH6, that produced daidzein and genistein from daidzin and genistin. Both of these bacteria have glucosidase activity. Strain HGH6 also reduces daidzein and genistein to dihydrodaidzein and dihydrogenistein, respectively, under anaerobic conditions. The reduction of the double bond between $\mathrm{C} 2$ and $\mathrm{C} 3$ in daidzein and genistein is isoflavone-specific; strain HGH6 does not reduce similar bonds in the flavonoids apigenin and chrysin [48]. Although dihydrodaidzein is an intermediate in the conversion of dihydrodaidzein to equol, no further metabolites were produced in cultures incubated with daidzein or synthetic dihydrodaidzein and dihydrogenistein, indicating that different enzymes and bacteria were required for this process [48].

Hur et al. [90] also isolated an anaerobic Gram-positive bacterium, designated as HGH136, by screening human fecal bacteria for daidzein-metabolizing strains. 16S rRNA analysis identified strain HGH136 to be closely related to Clostridium species [48]. This bacterium cleaves the C-ring of daidzein to produce $O$-DMA under anaerobic conditions [90]. $O$-DMA is also produced by the incubation of strain HGH136 with synthetic dihydrodaidzein [90]. No other metabolites were found as the result of incubation of strain HGH136 with either daidzein or dihydrodaidzein [90]. 
Later, another strain of Clostridium-like bacteria, designated TM-40, was found by Tamura et al. [91] to metabolize daidzin. The strain, which was isolated from the fecal material of a healthy young boy on a Japanese diet, is capable of converting both daidzin and daidzein to dihydrodaidzein. The 16S rRNA sequence of this strain is somewhat similar to Clostridium species but has $93 \%$ similarity to that of Coprobacillus catenaformis AB030218 [91]. Strain TM-40 enhanced the production of dihydrodaidzein in in vitro fecal cultures from equol producers and nonproducers, and enhanced the amount of equol produced in the cultures of producers [92].

\section{Genera of Equol-Producing Bacteria}

\subsection{Eggerthella, Adlercreutzia, and Asaccharobacter}

Among the intestinal colonic bacteria that are associated with production of equol are several strains of bacteria with sequence similarity to Eggerthella species. Maruo et al. [93] isolated nine strains of bacteria, with 16S rRNA genes having 93\% similarity to that of Eggerthella sinensis that are capable of metabolizing isoflavones by producing equol from daidzein. There were differences in biochemical reactions and cell wall peptidoglycan types between these strains and Eggerthella, so they were classified in a new genus and species as Adlercreutzia equolifaciens [93]. The type strain, A. equolifaciens DSM 19450, has been sequenced [93].

Wang et al. [94] isolated a strain, Julong 732, from human colonic bacteria, with $92.8 \%$ similarity to Eggerthella hongkongensis HKU10 and capable of converting dihydrodaidzein, but not daidzein, to equol. Through further experiments, this strain was shown to produce equol from synthetic $(3 S, 4 R)$-tetrahydrodaidzein by a tetrahydrodaidzein reductase, which is sensitive to oxygen and requires NADH for activity [95,96]. However, strain Julong 732 did not produce equol from dihydroequol, indicating that dihydroequol is not an intermediate in the production of equol from daidzein $[95,96]$.

Another strain of fecal bacteria, Eggerthella sp. strain YY7918, isolated from a human, converts daidzein and dihydrodaidzein to $S$-equol [97]. The genome of this strain has been sequenced [98].

An additional strain of bacteria, designated do03T, with similarity to Eggerthella and capable of producing equol from daidzein through production of dihydrodaidzein, has been isolated from rat intestine [99]. Butyric acid and arginine enhance the capability of strain do03T to produce equol in a more than four-fold quantity [99]. Due to distinctive biochemical characteristics, it was described as Asaccharobacter celatus [99].

Recently, some equol-producing bacteria isolated from stinky tofu have been shown by 16S rRNA analysis to have similarities to Eggerthella sinensis HKU14 and Eggerthella lenta JCM9979 [100,101].

\subsection{Slackia}

Some strains of the genus Slackia also metabolize isoflavones [102,103]. A Gram-positive, non-sporeforming bacterial strain, designated NATTS, was isolated from the fecal bacteria of an equol-producing individual. It converted daidzein to equol in cultures containing sorbose as carbon source [102]. Using the 16S RNA sequence, the strain was determined to belong to Slackia [102]. Further study showed that the numbers of Slackia sp. similar to strain NATTS in feces of Japanese men were correlated with the concentration of equol in the sera of both prostate cancer patients and controls [104]. 
The genes converting daidzein to dihydrodaidzein, and dihydrodaidzein to equol, from this bacterium were cloned and characterized [103]. They encoded three contiguous open reading frames (Orf 1-3) [103]. Tsuji et al. [103] showed that daidzein was converted to dihydrodaidzein by a protein encoded by orf3, belonging to the NADH:flavin oxidoreductase family. Dihydrodaidzein is converted to cisand trans-tetrahydrodaidzein (THD) by a protein encoded by $\operatorname{orf} 2$, belonging to the short-chain dehydrogenase/reductase superfamily. cis and trans-THD are converted to equol by a protein encoded by orfl that has an amino acid sequence similar to that of succinate dehydrogenase [103].

A strain of Slackia isoflavoniconvertens detected among human colonic bacteria capable of metabolizing isoflavones converts daidzein to equol via the intermediate dihydrodaidzein [105]; the author showed that it transformed $80 \mu \mathrm{M}$ of daidzein to equol in $10 \mathrm{~h}$ under anaerobic conditions. This bacterium also converts genistein to dihydrogenistein, which is then partially transformed to 5-hydroxyequol [105]. Schröder et al. [106], using daidzein-induced cultures of S. isoflavoniconvertens, identified the proteins involved in daidzein metabolism. They concluded that daidzein reductase converted daidzein and genistein to dihydrodaidzein and dpihydrogenistein; and recombinant dihydrodaidzein reductase and tetrahydrodaidzein reductase reduce dihydrodaidzein to equol [106].

Jin et al. [107] isolated a strain of bacteria, designated as DZE, capable of converting daidzein to equol and found that it had $89 \%$ and $88 \%$ similarity to Slackia faecicanis CCUG 48399 and Slackia exigua AF101240, respectively, and 87\% similarity to Slackia heliotrinireducens. They reported that strain DZE converted $150 \mu \mathrm{M}$ of daidzein to equol in $24 \mathrm{~h}$ with a $85 \%$ yield and transformed genistein to 5-hydroxyequol [108]. The strain has been named Slackia equolifaciens [108].

\subsection{Lactococcus}

Uchiyama et al. [109] isolated an equol-producing strain 20-92 from the human intestinal tract and identified it as Lactococcus garvieae by $16 \mathrm{~S}$ rRNA analysis. Genetic analysis has shown that this bacterium has an open reading frame, Orf US2, which encodes a dihydrodaidzein reductase that converts daidzein to dihydrodaidzein. This enzyme requires $\mathrm{NAD}(\mathrm{P}) \mathrm{H}$ for activity, is similar to the 3-oxoacyl-acyl-carrier-protein reductases of several bacteria, and belongs to the short-chain dehydrogenase/reductase family [110-112]. L. garvieae strain 20-92 also has a second open reading frame, orf-US3, which encodes tetrahydrodaidzein reductase (THDR) and converts dihydrodaidzein to tetrahydrodaidzein. THDR is similar to several putative fumarate reductase/succinate dehydrogenase flavoprotein domain proteins [110-112]. This strain also carries a novel dihydrodaidzein racemase capable of converting both the $(R)$ - and $(S)$-enantiomers of dihydrodaidzein to produce the racemate [110-112]. Shimada et al. [112] conclude that the equol biosynthesis pathway in L. garvieae strain 20-92 includes the following steps: L-Daidzein reductase converts daidzein to $(R)$-dihydrodaidzein. A novel dihydrodaidzein racemase rapidly racemizes the $(R)$-dihydrodaidzein and produces $(S)$-dihydrodaidzein. The resulting $(S)$-dihydrodaidzein is then preferentially converted to trans-tetrahydrodaidzein by dihydrodaidzein reductase. In the final reaction, L-tetrahydrodaidzein reductase converts trans-tetrahydrodaidzein to (S)-equol. L. garvieae also has been isolated from Italian cheese and appears to be an efficient equol producer [113]. L. garvieae has been investigated for use in producing equol-containing compounds that have been tested in experimental animals and humans [34,114]. 


\subsection{Other Isoflavone-Metabolizing Genera}

Raimondi et al. [49] screened 22 strains of Bifidobacterium representative of human strains and found that 12 of them efficiently converted daidzin to daidzein. However, none of the strains could convert daidzein to equol. Bifidobacterium animalis, B. longum-a, and B. pseudolongum deconjugate malonyl-, acetyl- and $\beta$-glucoside conjugates of daidzin, which are found in soy milk, to produce daidzein. These strains are also reported to transform daidzein to equol in soy milk [115].

A mixture containing Lactobacillus mucosae EPI2, Enterococcus faecium EPI1, Finegoldia magna EPI3, and Veillonella sp. strain EP, produced equol from daidzein, but none of the strains produced equol in pure culture [60].

\section{Factors Affecting Production of Equol}

In humans, conversion of daidzin and daidzein to equol and $O$-DMA results from the enzymatic activities of specific bacteria from the intestinal tract [54]. The capacity to produce equol and $O$-DMA remains stable over time and may be under genetic control of the host [116]. Van de Merwe et al. [116] postulated that the host genetic characteristics are important in determining the resident fecal flora. Brown et al. [10] found that production of equol is developmentally regulated and that a higher proportion of infants consuming formula produced equol than of breast-fed infants [10]. In Japanese adults, the positive association of a diet containing fat with higher excretion of equol in urine has been shown [89]. Also, a higher frequency of equol producers has been observed among vegetarians, who are $\sim 4.25$ times more likely to be $S$-equol producers than nonvegetarians [66].

Fruit consumption also may contribute to the enrichment of bacterial populations metabolizing daidzein. In a limited study, we showed that fecal bacterial samples from five individuals consuming soy diets varied in the metabolism of daidzein. After consumption of fruits for several months, samples from one in five subjects produced equol and $O$-DMA, and samples from two subjects produced either equol and dihydrodaidzein or equol and $O$-DMA [8]. This observation merits further investigation.

Use of antimicrobial agents also has been shown to affect in vivo and in vitro metabolism of daidzein and production of equol $[65,66]$. Sutherland et al. [117] showed that antimicrobial agents from different classes had different effects on the in vitro metabolism of daidzein by fecal bacteria from monkeys. Exposure of fecal bacteria to ceftriaxone did not affect metabolism of daidzein by the cultures. Bacteria metabolizing daidzein were eliminated after exposure to tetracycline. Treatment of cultures with ciprofloxacin enriched for the bacteria metabolizing daidzein. Cultures of fecal bacteria from monkeys treated with $4 \mu \mathrm{g} / \mathrm{mL}$ of ciprofloxacin metabolized more daidzein than untreated cultures. Brown et al. [10] also have shown that the use of antimicrobial agents affects the production of equol in human subjects. An antimicrobial susceptibility assay indicated that Eggerthella sp. strain YY7918, isolated from a human, that converts daidzein and dihydrodaidzein to $S$-equol, was susceptible to aminoglycoside, tetracycline, and new quinolone antibiotics [97]. Other factors affecting daidzein metabolism are short chain fatty acids and arginine. Both butyric acid and arginine enhanced the capability of Asaccharobacter celatus to metabolize daidzein by more than four-fold [99]. Age and unknown factors also may affect the metabolism of isoflavones. Recently, Franke et al. have shown inconsistencies in the production of equol over time in women, regardless of menopausal status [118,119]. 


\section{Metabolic Response to Consumption of Dietary Isoflavonoids}

Using nuclear magnetic resonance (NMR)-based metabolomics [120,121], the metabolites in the plasma and urine of subjects consuming soy diets containing isoflavones were analyzed. Consumption of soy resulted in altered levels of trimethylamine $N$-oxide, trimethylamine, choline, creatine, glutamine, and others. The levels of very low density lipoprotein, low density lipoprotein, and ketone bodies (acetate, acetoacetate, and hydroxybutyrate) relative to sugar concentration increased. Other chemicals related to lipid metabolism and biosynthesis also increased, indicating a soy consumption effect on the lipoprotein profile [120,121].

Decreases in plasma sugar, increases in lactate, and variations in the plasma levels of glucogenic amino acids (isoleucine and valine) were also observed. The levels of triglycerides were also altered in different subjects consuming soy or daidzein $(120,121]$ indicating changes in carbohydrate and energy metabolism resulting from soy intervention. Solanky et al. hypothesized that the increase in lactate and glucogenic amino acids may indicate inhibition of gluconeogenesis [120,121]. They concluded that isoflavones had inhibitory effects on glycolysis, resulting in a shift from carbohydrate to lipid metabolism. The response was subject-specific, and the authors indicated that variation in the colonic microflora and genetic variability influenced the effect of consumption of soy products. Administration of daidzein to subjects with different estrogen receptor genotypes decreased the levels of triglycerides and uric acid in serum to different extents [122]. The contribution of isoflavones from soy in alteration of plasma components, which could influence hormonal activities, is confounded by the fact that soy products contain other components that also may affect the plasma profile [120,121].

\section{Conclusions}

The incidence of a variety of hormone-dependent diseases, including coronary heart disease, atherosclerosis, and menopausal symptoms, is lower in the geographic regions where the population consumes a soy diet. The isoflavones in the soy have similarities to natural estradiols, which has generated interest in consumption of soy-related products for the prevention or alleviation of hormone-related symptoms, including menopause, in women. However, diets containing these compounds have variable results in different subjects, both in the effects and the types of metabolites produced in the individuals. There is a consensus that the differences in the effects for various individuals result from differences in the microfloras of the subjects taking isoflavones. The enzymatic activities of intestinal bacteria are involved in the metabolism, bioavailability, and conversion of isoflavones to compounds with altered estrogenic activities. Substantial research is in progress to find bacteria that produce equol from daidzein, because equol has a higher affinity for the estrogen receptors and a higher biological activity than daidzein. The identification of bacteria converting daidzein to equol should facilitate in vitro production of $S$-equol in quantities that could exert biological effects and aid in metabolomic studies using equol.

\section{Acknowledgments}

The authors thank John B. Sutherland and Kidon Sung for reviewing the manuscript and Carl E. Cerniglia for his research support. The views presented in this article do not necessarily reflect those of the U. S. Food and Drug Administration. 


\section{Conflict of Interest}

The author declare no conflicts of interest.

\section{References}

1. Hao, W.L.; Lee, Y.K. Microflora of the gastrointestinal tract: A review. Methods Mol. Biol. 2004, 268, 491-502.

2. Gerritsen, J.; Smidt, H.; Rijkers, G.T.; de Vos, W.M. Intestinal microbiota in human health and disease: The impact of probiotics. Genes Nutr. 2011, 6, 209-240.

3. Adlercreutz, H. Evolution, nutrition, intestinal microflora, and prevention of cancer: A hypothesis. Proc. Soc. Exp. Biol. Med. 1998, 217, 241-246.

4. Selkrig, J.; Wong, P.; Zhang, X.; Pettersson, S. Metabolic tinkering by the gut microbiome: Implications for brain development and function. Gut Microbes 2014, 5, 369-380.

5. Davis, C.D.; Milner, J.A. Gastrointestinal microflora, food components and colon cancer prevention. J. Nutr. Biochem. 2009, 20, 743-752.

6. Nicholson, J.K.; Holmes, E.; Lindon, J.C.; Wilson, I.D. The challenges of modeling mammalian biocomplexity. Nat. Biotechnol. 2004, 22, 1268-1274.

7. Joannou, G.E.; Kelly, G.E.; Reeder, A.Y.; Waring, M.; Nelson, C. A urinary profile study of dietary phytoestrogens. The identification and mode of metabolism of new isoflavonoids. J. Steroid Biochem. Mol. Biol. 1995, 54, 167-184.

8. Rafii, F.; Davis, C.; Park, M.; Heinze, T.M.; Beger, R.D. Variations in metabolism of the soy isoflavonoid daidzein by human intestinal microfloras from different individuals. Arch. Microbiol. 2003, 180, 11-16.

9. Yuan, J.P.; Wang, J.H.; Liu, X. Metabolism of dietary soy isoflavones to equol by human intestinal microflora-Implications for health. Mol. Nutr. Food Res. 2007, 51, 765-781.

10. Brown, N.M.; Galandi, S.L.; Summer, S.S.; Zhao, X.; Heubi, J.E.; King, E.C.; Setchell, K.D. $S$-(-)-Equol production is developmentally regulated and related to early diet composition. Nutr. Res. 2014, 34, 401-409.

11. Messina, M.; Watanabe, S.; Setchell, K.D. Report on the 8th international symposium on the role of soy in health promotion and chronic disease prevention and treatment. J. Nutr. 2009, 139, 796S-802S.

12. Setchell, K.D. Phytoestrogens: The biochemistry, physiology, and implications for human health of soy isoflavones. Am. J. Clin. Nutr. 1998, 68, 1333S-1346S.

13. Setchell, K.D.; Cassidy, A. Dietary isoflavones: Biological effects and relevance to human health. J. Nutr. 1999, 129, 758S-767S.

14. Setchell, K.D.; Brown, N.M.; Desai, P.B.; Zimmer-Nechimias, L.; Wolfe, B.; Jakate, A.S.; Creutzinger, V.; Heubi, J.E. Bioavailability, disposition, and dose-response effects of soy isoflavones when consumed by healthy women at physiologically typical dietary intakes. J. Nutr. 2003, 133, 1027-1035.

15. Setchell, K.D.; Zhao, X.; Shoaf, S.E.; Ragland, K. The pharmacokinetics of $S$-(-)-equol administered as SE5-OH tablets to healthy postmenopausal women. J. Nutr. 2009, 139, 2037-2043. 
16. Lampe, J.W.; Karr, S.C.; Hutchins, A.M.; Slavin, J.L. Urinary equol excretion with a soy challenge: Influence of habitual diet. Proc. Soc. Exp. Biol. Med. 1998, 217, 335-339.

17. Rowland, I.; Wiseman, H.; Sanders, T.; Adlercreutz, H.; Bowey, E. Metabolism of oestrogens and phytoestrogens: Role of the gut microflora. Biochem. Soc. Trans. 1999, 27, 304-308.

18. Rowland, I.; Faughnan, M.; Hoey, L.; Wahala, K.; Williamson, G.; Cassidy, A. Bioavailability of phyto-oestrogens. Br. J. Nutr. 2003, 89 (Suppl. 1), S45-S58.

19. Xu, L.; Du, B.; Xu, B. A systematic, comparative study on the beneficial health components and antioxidant activities of commercially fermented soy products marketed in china. Food Chem 2015, 174, 202-213.

20. Munro, I.C.; Harwood, M.; Hlywka, J.J.; Stephen, A.M.; Doull, J.; Flamm, W.G.; Adlercreutz, H. Soy isoflavones: A safety review. Nutr. Rev. 2003, 61, 1-33.

21. Wang, H.; Murphy, P.A.Isoflavone composition of American and Japanese soybeans in Iowa: Effects of variety, crop year, and location. J. Agric. Food Chem. 1994, 42, 1674-1677.

22. Hur, H.; Rafii, F. Biotransformation of the isoflavonoids biochanin A, formononetin, and glycitein by Eubacterium limosum. FEMS Microbiol. Lett. 2000, 192, 21-25.

23. Jackson, R.L.; Greiwe, J.S.; Desai, P.B.; Schwen, R.J. Single-dose and steady-state pharmacokinetic studies of $S$-equol, a potent nonhormonal, estrogen receptor beta-agonist being developed for the treatment of menopausal symptoms. Menopause 2011, 18, 185-193.

24. Jackson, R.L.; Greiwe, J.S.; Schwen, R.J. Ageing skin: Oestrogen receptor beta agonists offer an approach to change the outcome. Exp. Dermatol. 2011, 20, 879-882.

25. Lehmann, L.; Esch, H.L.; Wagner, J.; Rohnstock, L.; Metzler, M. Estrogenic and genotoxic potential of equol and two hydroxylated metabolites of daidzein in cultured human Ishikawa cells. Toxicol. Lett. 2005, 158, 72-86.

26. Chiang, S.S.; Pan, T.M. Beneficial effects of phytoestrogens and their metabolites produced by intestinal microflora on bone health. Appl. Microbiol. Biotechnol. 2013, 97, 1489-1500.

27. Ju, Y.H.; Fultz, J.; Allred, K.F.; Doerge, D.R.; Helferich, W.G. Effects of dietary daidzein and its metabolite, equol, at physiological concentrations on the growth of estrogen-dependent human breast cancer (MCF-7) tumors implanted in ovariectomized athymic mice. Carcinogenesis 2006, 27, 856-863.

28. Sugiyama, A.; Shitan, N.; Yazaki, K. Involvement of a soybean ATP-binding cassette-type transporter in the secretion of genistein, a signal flavonoid in legume-rhizobium symbiosis. Plant Physiol. 2007, 144, 2000-2008.

29. Patisaul, H.B.; Jefferson, W. The pros and cons of phytoestrogens. Front. Neuroendocrinol. 2010, $31,400-419$.

30. Wiseman, H. The therapeutic potential of phytoestrogens. Expert Opin. Investig. Drugs 2000, 9, 1829-1840.

31. Atkinson, C.; Frankenfeld, C.L.; Lampe, J.W. Gut bacterial metabolism of the soy isoflavone daidzein: Exploring the relevance to human health. Exp. Biol. Med. (Maywood) 2005, 230, 155-170.

32. Aso, T.; Uchiyama, S.; Matsumura, Y.; Taguchi, M.; Nozaki, M.; Takamatsu, K.; Ishizuka, B.; Kubota, T.; Mizunuma, H.; Ohta, H. A natural $S$-equol supplement alleviates hot flushes and other menopausal symptoms in equol nonproducing postmenopausal Japanese women. J. Women's Health (Larchmt.) 2012, 21, 92-100. 
33. Crawford, S.L.; Jackson, E.A.; Churchill, L.; Lampe, J.W.; Leung, K.; Ockene, J.K. Impact of dose, frequency of administration, and equol production on efficacy of isoflavones for menopausal hot flashes: A pilot randomized trial. Menopause 2013, 20, 936-945.

34. Jenks, B.H.; Iwashita, S.; Nakagawa, Y.; Ragland, K.; Lee, J.; Carson, W.H.; Ueno, T.; Uchiyama, S. A pilot study on the effects of $S$-equol compared to soy isoflavones on menopausal hot flash frequency. J. Women's Health (Larchmt.) 2012, 21, 674-682.

35. DellaPenna, D. Nutritional genomics: Manipulating plant micronutrients to improve human health. Science 1999, 285, 375-379.

36. Setchell, K.D.; Brown, N.M.; Lydeking-Olsen, E. The clinical importance of the metabolite equol-A clue to the effectiveness of soy and its isoflavones. J. Nutr. 2002, 132, 3577-3584.

37. Khaodhiar, L.; Ricciotti, H.A.; Li, L.; Pan, W.; Schickel, M.; Zhou, J.; Blackburn, G.L. Daidzein-rich isoflavone aglycones are potentially effective in reducing hot flashes in menopausal women. Menopause 2008, 15, 125-132.

38. Morton, M.S.; Arisaka, O.; Miyake, N.; Morgan, L.D.; Evans, B.A. Phytoestrogen concentrations in serum from Japanese men and women over forty years of age. J. Nutr. 2002, 132, 3168-3171.

39. Cornwell, T.; Cohick, W.; Raskin, I. Dietary phytoestrogens and health. Phytochemistry 2004, 65, 995-1016.

40. Loutchanwoot, P.; Srivilai, P.; Jarry, H. Effects of the natural endocrine disruptor equol on the pituitary function in adult male rats. Toxicology 2013, 304, 69-75.

41. Lund, T.D.; Blake, C.; Bu, L.; Hamaker, A.N.; Lephart, E.D. Equol, an isoflavonoid: Potential for improved prostate health, in vitro and in vivo evidence. Reprod. Biol. Endocrinol. 2011, 9, 4.

42. Marino, M.; Galluzzo, P.; Ascenzi, P. Estrogen signaling multiple pathways to impact gene transcription. Curr. Genomics 2006, 7, 497-508.

43. Neese, S.L.; Pisani, S.L.; Doerge, D.R.; Helferich, W.G.; Sepehr, E.; Chittiboyina, A.G.; Rotte, S.C.; Smillie, T.J.; Khan, I.A.; Korol, D.L.; et al. The effects of dietary treatment with $S$-equol on learning and memory processes in middle-aged ovariectomized rats. Neurotoxicol. Teratol. 2014, 41, 80-88.

44. Oyama, A.; Ueno, T.; Uchiyama, S.; Aihara, T.; Miyake, A.; Kondo, S.; Matsunaga, K. The effects of natural $S$-equol supplementation on skin aging in postmenopausal women: A pilot randomized placebo-controlled trial. Menopause 2012, 19, 202-210.

45. Takeuchi, S.; Takahashi, T.; Sawada, Y.; Iida, M.; Matsuda, T.; Kojima, H. Comparative study on the nuclear hormone receptor activity of various phytochemicals and their metabolites by reporter gene assays using Chinese hamster ovary cells. Biol. Pharm. Bull. 2009, 32, 195-202.

46. Uehara, M. Isoflavone metabolism and bone-sparing effects of daidzein-metabolites. J. Clin. Biochem. Nutr. 2013, 52, 193-201.

47. Yao, J.; Zhao, L.; Mao, Z.; Chen, S.; Wong, K.C.; To, J.; Brinton, R.D. Potentiation of brain mitochondrial function by $S$-equol and $R / S$-equol estrogen receptor $\beta$-selective phytoSERM treatments. Brain Res. 2013, 1514, 128-141.

48. Hur, H.G.; Lay, J.O., Jr.; Beger, R.D.; Freeman, J.P.; Rafii, F. Isolation of human intestinal bacteria metabolizing the natural isoflavone glycosides daidzin and genistin. Arch. Microbiol. 2000, 174, $422-428$. 
49. Raimondi, S.; Roncaglia, L.; De Lucia, M.; Amaretti, A.; Leonardi, A.; Pagnoni, U.M.; Rossi, M. Bioconversion of soy isoflavones daidzin and daidzein by Bifidobacterium strains. Appl. Microbiol. Biotechnol. 2009, 81, 943-950.

50. Rossi, M.; Amaretti, A.; Leonardi, A.; Raimondi, S.; Simone, M.; Quartieri, A. Potential impact of probiotic consumption on the bioactivity of dietary phytochemicals. J. Agric. Food Chem. 2013, 61, 9551-9558.

51. Morito, K.; Hirose, T.; Kinjo, J.; Hirakawa, T.; Okawa, M.; Nohara, T.; Ogawa, S.; Inoue, S.; Muramatsu, M.; Masamune, Y. Interaction of phytoestrogens with estrogen receptors alpha and beta. Biol. Pharm. Bull. 2001, 24, 351-356.

52. Richelle, M.; Pridmore-Merten, S.; Bodenstab, S.; Enslen, M.; Offord, E.A. Hydrolysis of isoflavone glycosides to aglycones by beta-glycosidase does not alter plasma and urine isoflavone pharmacokinetics in postmenopausal women. J. Nutr. 2002, 132, 2587-2592.

53. Zubik, L.; Meydani, M. Bioavailability of soybean isoflavones from aglycone and glucoside forms in American women. Am. J. Clin. Nutr. 2003, 77, 1459-1465.

54. Setchell, K.D.; Clerici, C. Equol: History, chemistry, and formation. J. Nutr. 2010, 140, $1355 \mathrm{~S}-1362 \mathrm{~S}$.

55. Setchell, K.D.; Clerici, C. Equol: Pharmacokinetics and biological actions. J. Nutr. 2010, 140, 1363S-1368S.

56. Day, A.J.; DuPont, M.S.; Ridley, S.; Rhodes, M.; Rhodes, M.J.; Morgan, M.R.; Williamson, G. Deglycosylation of flavonoid and isoflavonoid glycosides by human small intestine and liver beta-glucosidase activity. FEBS Lett. 1998, 436, 71-75.

57. Gu, L.; House, S.E.; Prior, R.L.; Fang, N.; Ronis, M.J.; Clarkson, T.B.; Wilson, M.E.; Badger, T.M. Metabolic phenotype of isoflavones differ among female rats, pigs, monkeys, and women. J. Nutr. 2006, 136, 1215-1221.

58. Chang, Y.C.; Nair, M.G. Metabolism of daidzein and genistein by intestinal bacteria. J. Nat. Prod. 1995, 58, 1892-1896.

59. Chang, Y.C.; Nair, M.G.; Nitiss, J.L. Metabolites of daidzein and genistein and their biological activities. J. Nat. Prod. 1995, 58, 1901-1905.

60. Decroos, K.; Vanhemmens, S.; Cattoir, S.; Boon, N.; Verstraete, W. Isolation and characterisation of an equol-producing mixed microbial culture from a human faecal sample and its activity under gastrointestinal conditions. Arch. Microbiol. 2005, 183, 45-55.

61. Gardana, C.; Canzi, E.; Simonetti, P. The role of diet in the metabolism of daidzein by human faecal microbiota sampled from Italian volunteers. J. Nutr. Biochem. 2009, 20, 940-947.

62. Gardana, C.; Canzi, E.; Simonetti, P. $R(-)-O$-Desmethylangolensin is the main enantiomeric form of daidzein metabolite produced by human in vitro and in vivo. J. Chromatogr. B Anal. Technol. Biomed. Life Sci. 2014, 953-954, 30-37.

63. Harris, R.M.; Wood, D.M.; Bottomley, L.; Blagg, S.; Owen, K.; Hughes, P.J.; Waring, R.H.; Kirk, C.J. Phytoestrogens are potent inhibitors of estrogen sulfation: Implications for breast cancer risk and treatment. J. Clin. Endocrinol. MeTable 2004, 89, 1779-1787.

64. Axelson, M.; Sjovall, J.; Gustafsson, B.E.; Setchell, K.D. Soya-A dietary source of the non-steroidal oestrogen equol in man and animals. J. Endocrinol. 1984, 102, 49-56. 
65. Atkinson, C.; Berman, S.; Humbert, O.; Lampe, J.W. In vitro incubation of human feces with daidzein and antibiotics suggests interindividual differences in the bacteria responsible for equol production. J. Nutr. 2004, 134, 596-599.

66. Setchell, K.D.; Cole, S.J. Method of defining equol-producer status and its frequency among vegetarians. J. Nutr. 2006, 136, 2188-2193.

67. Klinge, C.M. Estrogen receptor interaction with estrogen response elements. Nucl. Acids Res. 2001, 29, 2905-2919.

68. Hirvonen, J.; Rajalin, A.M.; Wohlfahrt, G.; Adlercreutz, H.; Wahala, K.; Aarnisalo, P. Transcriptional activity of estrogen-related receptor gamma (ERRgamma) is stimulated by the phytoestrogen equol. J. Steroid Biochem. Mol. Biol. 2011, 123, 46-57.

69. Magee, P.J. Is equol production beneficial to health? Proc. Nutr. Soc. 2011, 70, 10-18.

70. Magee, P.J.; Allsopp, P.; Samaletdin, A.; Rowland, I.R. Daidzein, $R$-(+)-Equol and $S$-(-)-equol inhibit the invasion of MDA-MB-231 breast cancer cells potentially via the down-regulation of matrix metalloproteinase-2. Eur. J. Nutr. 2014, 53, 345-350.

71. van der Velpen, V.; Geelen, A.; Schouten, E.G.; Hollman, P.C.; Afman, L.A.; van’t Veer, P. Estrogen receptor-mediated effects of isoflavone supplementation were not observed in whole-genome gene expression profiles of peripheral blood mononuclear cells in postmenopausal, equol-producing women. J. Nutr. 2013, 143, 774-780.

72. Kelly, L.A.; Seidlova-Wuttke, D.; Wuttke, W.; O’Leary, J.J.; Norris, L.A. Estrogen receptor alpha augments changes in hemostatic gene expression in HepG2 cells treated with estradiol and phytoestrogens. Phytomedicine 2014, 21, 155-158.

73. Choi, E.J.; Kim, G.H. The antioxidant activity of daidzein metabolites, $O$-desmethylangolensin and equol, in HepG2 cells. Mol. Med. Rep. 2014, 9, 328-332.

74. Del Rio, D.; Rodriguez-Mateos, A.; Spencer, J.P.; Tognolini, M.; Borges, G.; Crozier, A. Dietary (poly)phenolics in human health: Structures, bioavailability, and evidence of protective effects against chronic diseases. Antioxid. Redox Signal. 2013, 18, 1818-1892.

75. Jackman, K.A.; Woodman, O.L.; Sobey, C.G. Isoflavones, equol and cardiovascular disease: Pharmacological and therapeutic insights. Curr. Med. Chem. 2007, 14, 2824-2830.

76. Mahmoud, A.M.; Yang, W.; Bosland, M.C. Soy isoflavones and prostate cancer: A review of molecular mechanisms. J. Steroid Biochem. Mol. Biol. 2014, 140, 116-132.

77. Wei, X.J.; Wu, J.; Ni, Y.D.; Lu, L.Z.; Zhao, R.Q. Antioxidant effect of a phytoestrogen equol on cultured muscle cells of embryonic broilers. In Vitro Cell Dev. Biol. Anim. 2011, 47, 735-741.

78. Markiewicz, L.; Garey, J.; Adlercreutz, H.; Gurpide, E. In vitro bioassays of non-steroidal phytoestrogens. J. Steroid Biochem. Mol. Biol. 1993, 45, 399-405.

79. Shutt, D.A.; Cox, R.I. Steroid and phyto-oestrogen binding to sheep uterine receptors in vitro. J. Endocrinol. 1972, 52, 299-310.

80. Sathyamoorthy, N.; Wang, T.T. Differential effects of dietary phyto-oestrogens daidzein and equol on human breast cancer MCF-7 cells. Eur. J. Cancer 1997, 33, 2384-2389.

81. Kostelac, D.; Rechkemmer, G.; Briviba, K. Phytoestrogens modulate binding response of estrogen receptors alpha and beta to the estrogen response element. J. Agric. Food Chem. 2003, 51, 7632-7635. 
82. Vedavanam, K.; Srijayanta, S.; O’Reilly, J.; Raman, A.; Wiseman, H. Antioxidant action and potential antidiabetic properties of an isoflavonoid-containing soyabean phytochemical extract (SPE). Phytother. Res. 1999, 13, 601-608.

83. Rimbach, G.; De Pascual-Teresa, S.; Ewins, B.A.; Matsugo, S.; Uchida, Y.; Minihane, A.M.; Turner, R.; VafeiAdou, K.; Weinberg, P.D. Antioxidant and free radical scavenging activity of isoflavone metabolites. Xenobiotica 2003, 33, 913-925.

84. Hwang, J.; Wang, J.; Morazzoni, P.; Hodis, H.N.; Sevanian, A. The phytoestrogen equol increases nitric oxide availability by inhibiting superoxide production: An antioxidant mechanism for cell-mediated ldl modification. Free Radic. Biol. Med. 2003, 34, 1271-1282.

85. Froyen, E.B.; Steinberg, F.M. Soy isoflavones increase quinone reductase in hepa-1c1c7 cells via estrogen receptor beta and nuclear factor erythroid 2-related factor 2 binding to the antioxidant response element. J. Nutr. Biochem. 2011, 22, 843-848.

86. Hedlund, T.E.; Johannes, W.U.; Miller, G.J. Soy isoflavonoid equol modulates the growth of benign and malignant prostatic epithelial cells in vitro. Prostate 2003, 54, 68-78.

87. Dewi, F.N.; Wood, C.E.; Lampe, J.W.; Hullar, M.A.; Franke, A.A.; Golden, D.L.; Adams, M.R.; Cline, J.M. Endogenous and exogenous equol are antiestrogenic in reproductive tissues of apolipoprotein E-null mice. J. Nutr. 2012, 142, 1829-1835.

88. Bowey, E.; Adlercreutz, H.; Rowland, I. Metabolism of isoflavones and lignans by the gut microflora: A study in germ-free and human flora associated rats. Food Chem. Toxicol. 2003, 41, 631-636.

89. Rowland, I.R.; Wiseman, H.; Sanders, T.A.; Adlercreutz, H.; Bowey, E.A. Interindividual variation in metabolism of soy isoflavones and lignans: Influence of habitual diet on equol production by the gut microflora. Nutr. Cancer 2000, 36, 27-32.

90. Hur, H.G.; Beger, R.D.; Heinze, T.M.; Lay, J.O., Jr.; Freeman, J.P.; Dore, J.; Rafii, F. Isolation of an anaerobic intestinal bacterium capable of cleaving the C-ring of the isoflavonoid daidzein. Arch. Microbiol. 2002, 178, 8-12.

91. Tamura, M.; Tsushida, T.; Shinohara, K. Isolation of an isoflavone-metabolizing, Clostridium-like bacterium, strain TM-40, from human faeces. Anaerobe 2007, 13, 32-35.

92. Tamura, M.; Hori, S.; Nakagawa, H. Dihydrodaidzein-producing clostridium-like intestinal bacterium, strain TM-40, affects in vitro metabolism of daidzein by fecal microbiota of human male equol producer and non-producers. Biosci. Microflora 2011, 30, 65-71.

93. Maruo, T.; Sakamoto, M.; Ito, C.; Toda, T.; Benno, Y. Adlercreutzia equolifaciens gen. nov., sp. nov., an equol-producing bacterium isolated from human faeces, and emended description of the genus Eggerthella. Int. J. Syst. Evol. Microbiol. 2008, 58, 1221-1227.

94. Wang, X.L.; Hur, H.G.; Lee, J.H.; Kim, K.T.; Kim, S.I. Enantioselective synthesis of $S$-equol from dihydrodaidzein by a newly isolated anaerobic human intestinal bacterium. Appl. Environ. Microbiol. 2005, 71, 214-219.

95. Kim, M.; Kim, S.I.; Han, J.; Wang, X.L.; Song, D.G.; Kim, S.U. Stereospecific biotransformation of dihydrodaidzein into (3S)-equol by the human intestinal bacterium Eggerthella strain Julong 732. Appl. Environ. Microbiol. 2009, 75, 3062-3068. 
96. Kim, M.; Marsh, E.N.; Kim, S.U.; Han, J. Conversion of (3S,4R)-tetrahydrodaidzein to (3S)-equol by THD reductase: Proposed mechanism involving a radical intermediate. Biochemistry 2010, 49, 5582-5587.

97. Yokoyama, S.; Suzuki, T. Isolation and characterization of a novel equol-producing bacterium from human feces. Biosci. Biotechnol. Biochem. 2008, 72, 2660-2666.

98. Yokoyama, S.; Oshima, K.; Nomura, I.; Hattori, M.; Suzuki, T. Complete genomic sequence of the equol-producing bacterium Eggerthella sp. strain YY7918, isolated from adult human intestine. J. Bacteriol. 2011, 193, 5570-5571.

99. Minamida, K.; Ota, K.; Nishimukai, M.; Tanaka, M.; Abe, A.; Sone, T.; Tomita, F.; Hara, H.; Asano, K. Asaccharobacter celatus gen. nov., sp. nov., isolated from rat caecum. Int. J. Syst. Evol. Microbiol. 2008, 58, 1238-1240.

100. Abiru, Y.; Kumemura, M.; Ueno, T.; Uchiyama, S.; Masaki, K. Discovery of an $S$-equol rich food, stinky tofu, a traditional fermented soy product in Taiwan. Int. J. Food Sci. Nutr. 2012, 63, 964-970.

101. Abiru, Y.; Ueno, T.; Uchiyama, S. Isolation and characterization of novel $S$-equol-producing bacteria from brines of stinky tofu, a traditional fermented soy food in Taiwan. Int. J. Food Sci. Nutr. 2013, 64, 936-943.

102. Tsuji, H.; Moriyama, K.; Nomoto, K.; Miyanaga, N.; Akaza, H. Isolation and characterization of the equol-producing bacterium Slackia sp. strain NATTS. Arch. Microbiol. 2010, 192, 279-287.

103. Tsuji, H.; Moriyama, K.; Nomoto, K.; Akaza, H. Identification of an enzyme system for daidzein-to-equol conversion in Slackia sp. strain NATTS. Appl. Environ. Microbiol. 2012, 78, 1228-1236.

104. Sugiyama, Y.; Nagata, Y.; Fukuta, F.; Takayanagi, A.; Masumori, N.; Tsukamoto, T.; Akasaka, H.; Ohnishi, H.; Saito, S.; Miura, T.; Moriyama, K.; Tsuji, H.; Akaza, H.; Mori, M. Counts of Slackia sp. strain NATTS in intestinal flora are correlated to serum concentrations of equol both in prostate cancer cases and controls in Japanese men. Asian Pac. J. Cancer Prev. 2014, 15, 2693-2697.

105. Matthies, A.; Loh, G.; Blaut, M.; Braune, A. Daidzein and genistein are converted to equol and 5-hydroxyequol by human intestinal Slackia isoflavoniconvertens in gnotobiotic rats. J. Nutr. 2012, $142,40-46$.

106. Schröder, C.; Matthies, A.; Engst, W.; Blaut, M.; Braune, A. Identification and expression of genes involved in the conversion of daidzein and genistein by the equol-forming bacterium Slackia isoflavoniconvertens. Appl. Environ. Microbiol. 2013, 79, 3494-3502.

107. Jin, J.S.; Nishihata, T.; Kakiuchi, N.; Hattori, M. Biotransformation of C-glucosylisoflavone puerarin to estrogenic (3S)-equol in co-culture of two human intestinal bacteria. Biol. Pharm. Bull. 2008, 31, 1621-1625.

108. Jin, J.S.; Kitahara, M.; Sakamoto, M.; Hattori, M.; Benno, Y. Slackia equolifaciens sp. nov., a human intestinal bacterium capable of producing equol. Int. J. Syst. Evol. Microbiol. 2010, 60, 1721-1724.

109. Uchiyama, S.; Ueno, T.; Suzuki, T. Identification of a newly isolated equol-producing lactic acid bacterium from the human feces. J. Intest. Microbiol. 2007, 21, 217-220. 
110. Shimada, Y.; Yasuda, S.; Takahashi, M.; Hayashi, T.; Miyazawa, N.; Sato, I.; Abiru, Y.; Uchiyama, S.; Hishigaki, H. Cloning and expression of a novel NADP(H)-dependent daidzein reductase, an enzyme involved in the metabolism of daidzein, from equol-producing Lactococcus strain 20-92. Appl. Environ. Microbiol. 2010, 76, 5892-5901.

111. Shimada, Y.; Takahashi, M.; Miyazawa, N.; Ohtani, T.; Abiru, Y.; Uchiyama, S.; Hishigaki, H. Identification of two novel reductases involved in equol biosynthesis in Lactococcus strain 20-92. J. Mol. Microbiol. Biotechnol. 2011, 21, 160-172.

112. Shimada, Y.; Takahashi, M.; Miyazawa, N.; Abiru, Y.; Uchiyama, S.; Hishigaki, H. Identification of a novel dihydrodaidzein racemase essential for biosynthesis of equol from daidzein in Lactococcus sp. strain 20-92. Appl. Environ. Microbiol. 2012, 78, 4902-4907.

113. Fortina, M.G.; Ricci, G.; Foschino, R.; Picozzi, C.; Dolci, P.; Zeppa, G.; Cocolin, L.; Manachini, P.L. Phenotypic typing, technological properties and safety aspects of Lactococcus garvieae strains from dairy environments. J. Appl. Microbiol. 2007, 103, 445-453.

114. Matulka, R.A.; Matsuura, I.; Uesugi, T.; Ueno, T.; Burdock, G. Developmental and reproductive effects of SE5-OH: An equol-rich soy-based ingredient. J. Toxicol. 2009, 2009, 307618.

115. Tsangalis, D.; Ashton, J.F.; McGill, A.E.J.; Shah, N.P. Enzymic transformation of isoflavone phytoestrogens in soy milk by beta glucosidase producing bacteria. J. Food Sci. 2002, 67, 3104-3113.

116. Van de Merwe, J.P.; Stegeman, J.H.; Hazenberg, M.P. The resident faecal flora is determined by genetic characteristics of the host. Implications for Crohn's disease? Antonie Leeuwenhoek 1983, 49, 119-124.

117. Sutherland, J.B.; Bridges, B.M.; Heinze, T.M.; Adams, M.R.; Delio, P.J.; Hotchkiss, C.; Rafii, F. Comparison of the effects of antimicrobial agents from three different classes on metabolism of isoflavonoids by colonic microflora using Etest strips. Curr. Microbiol. 2012, 64, 60-65.

118. Franke, A.A.; Lai, J.F.; Pagano, I.; Morimoto, Y.; Maskarinec, G. Equol production changes over time in pre-menopausal women. Br. J. Nutr. 2012, 107, 1201-1206.

119. Franke, A.A.; Lai, J.F.; Halm, B.M. Absorption, distribution, metabolism, and excretion of isoflavonoids after soy intake. Arch. Biochem. Biophys. 2014, 559, 24-28.

120. Solanky, K.S.; Bailey, N.J.; Beckwith-Hall, B.M.; Bingham, S.; Davis, A.; Holmes, E.; Nicholson, J.K.; Cassidy, A. Biofluid ${ }^{1} \mathrm{H}$ NMR-based metabonomic techniques in nutrition research-Metabolic effects of dietary isoflavones in humans. J. Nutr. Biochem. 2005, 16, 236-244.

121. Solanky, K.S.; Bailey, N.J.; Beckwith-Hall, B.M.; Davis, A.; Bingham, S.; Holmes, E.; Nicholson, J.K.; Cassidy, A. Application of biofluid ${ }^{1} \mathrm{H}$ nuclear magnetic resonance-based metabonomic techniques for the analysis of the biochemical effects of dietary isoflavones on human plasma profile. Anal. Biochem. 2003, 323, 197-204.

122. Qin, Y.; Shu, F.; Zeng, Y.; Meng, X.; Wang, B.; Diao, L.; Wang, L.; Wan, J.; Zhu, J.; Wang, J.; et al. Daidzein supplementation decreases serum triglyceride111 and uric acid concentrations in hypercholesterolemic adults with the effect on triglycerides being greater in those with the GA compared with the GG genotype of Esr- $\beta$ rsai. J. Nutr. 2014, 144, 49-54.

(C) 2015 by the authors; licensee MDPI, Basel, Switzerland. This article is an open access article distributed under the terms and conditions of the Creative Commons Attribution license (http://creativecommons.org/licenses/by/4.0/). 